\title{
The growth and structure of short period (001) $\mathrm{Hg}_{1-x} \mathrm{Cd}_{x} \mathrm{Te}-\mathrm{HgTe}$ superlattices
}

\author{
C. R. Becker, L. He, ${ }^{\text {a) }}$ M. M. Regnet, M. M. Kraus, Y. S. Wu, and G. Landwehr \\ Physikalisches Institut der Universität Würzburg, Am Hubland, 97074 Würzburg, Germany \\ X. F. Zhang ${ }^{\text {b) }}$ and H. Zhang ${ }^{\text {) }}$ \\ Institut für Festkörperforschung, Forschungszentrum Jülich, 52425 Jülich, Germany
}

(Received 25 February 1993; accepted for publication 30 April 1993)

\begin{abstract}
Molecular beam epitaxially grown short period (001) $\mathrm{Hg}_{1-x} \mathrm{Cd}_{x} \mathrm{Te}-\mathrm{HgTe}$ superlattices have been systematically investigated. Several narrow well widths were chosen, e.g., 30,35 and $40 \AA$, and the barrier widths were varied between 24 and $90 \AA$ for a particular well width. Both the well width and the total period were determined directly by means of $\mathrm{x}$-ray diffraction. The well width was determined by exploiting the high reflectivity from $\mathrm{HgTe}$ and the low reflectivity from $\mathrm{CdTe}$ for the (002) Bragg reflection. Knowing the well and barrier widths we have been able to set an upper limit on the average Cd concentration of the barriers, $\bar{x}_{b}$, by annealing several superlattices and then measuring the composition of the resulting alloy. $\bar{x}_{b}$ was shown to decrease exponentially with decreasing barrier width. The structure of a very short period superlattice, i.e., $31.4 \AA$, was also investigated by transmission electron microscopy, corroborating the $\mathrm{x}$-ray diffraction results.
\end{abstract}

\section{INTRODUCTION}

The CdTe-HgTe superlattice was first proposed by Schulman and McGill in 1979. ${ }^{1}$ The actual realization of these superlattices by molecular beam epitaxy (MBE) was carried out by Faurie et al. in $1982 .{ }^{2}$ Thereafter numerous publications have dealt with the novel electrical and optical properties of these structures, which have been reviewed by Faurie, ${ }^{3}$ McGill et al. ${ }^{4}$ Meyer et al. ${ }^{5}$ and others.

The CdTe-HgTe superlattice has potential advantages in infrared applications compared to the alloy. For example, better control over the band gap of narrow gap superlattices has been predicted. ${ }^{6,7}$ The band gap is controlled primarily by the well thickness and to a lesser extent by the barrier thickness. It increases from approximately 10 to $200 \mathrm{meV}$ when the well width is reduced from 100 to $30 \AA$. In contrast the band gap of the alloy depends on its composition which has to be progressively better controlled as the band gap goes to zero. The band gap of a superlattice is a smooth, slowly varying function of the layer thicknesses and hence arguably easier to control. However the barrier normally consists of $\mathrm{Hg}_{1-x} \mathrm{Cd}_{x} \mathrm{Te}$ and not $\mathrm{CdTe}$ because the $\mathrm{Hg}$ flux is left on during growth and both $\mathrm{Hg}$ and $\mathrm{Cd}$ compete for cation sites. The composition of the well and the barrier influences the height of the potential barrier between the two and hence the band gap. Their $x$ values are not readily accessible but should depend primarily on their initial values, i.e., on the growth parameters, and in the case of narrow layers on subsequent interdiffusion. Kim et al. ${ }^{8}$ have shown that interdiffusion is two orders of magnitude larger near the surface when compared to a depth of $7000 \AA$ or more. Therefore interdiffusion

\footnotetext{
a) Permanent address: Shanghai Institute of Technical Physics, Chinese Academy of Sciences, Shanghai, People's Republic of China.

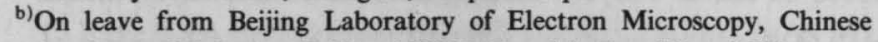
Academy of Sciences, Beijing 100080, People's Republic of China.

${ }^{c)}$ Present address: MSD-212 Argonne National Laboratory, 9700 South Cass Avenue, Argonne, Il 60439.
}

should depend primarily on how long a particular layer spends near the surface which is constant with the exception of the superlattice periods grown last.

The superlattice period is readily accessible by $\mathrm{x}$-ray diffraction experiments; however, the well and barrier thicknesses and their compositions are not so easily determined. Historically well and barrier thicknesses have been inferred from the growth parameters or measured by transmission electron microscopy (TEM).${ }^{9}$ We have been able to determine the well thickness and hence that of the barrier by means of high resolution x-ray diffraction. ${ }^{10-12}$ This is done by taking advantage of the large $\mathrm{HgTe}$ structure factor compared to that of CdTe for the (002) Bragg reflection. The well thickness as determined by this method has been corroborated by TEM for a superlattice with an extremely short period of $31.4 \AA$.

The composition of the well and barrier cannot be determined directly except possibly by a destructive method such as chemical mapping ${ }^{8}$ which utilizes TEM. In this investigation we have estimated the composition of the initial barrier material by means of transmission and reflection measurements on thick test layers of (001) $\mathrm{Hg}_{1-x} \mathrm{Cd}_{x} \mathrm{Te}$ grown under identical conditions with the exception of the presence of the HgTe wells. Knowing the well and barrier thicknesses we have been able to set an upper limit on the actual composition of the barriers by annealing several superlattices and then measuring the composition of the resulting alloy.

\section{EXPERIMENTAL AND THEORETICAL DETAILS}

\section{A. Growth}

Epitaxial growth was carried out in a four chamber RIBER 2300, molecular beam epitaxial (MBE) system which has been modified to permit the growth of $\mathrm{Hg}$ based materials. The vacuum in the growth chamber is better than $6 \times 10^{-10}$ Torr when no $\mathrm{Hg}$ has recently been admit- 
ted. Three MBE cells were employed, two of which were commercial cells and which contained high purity $\mathrm{CdTe}$ and Te. The third cell is a self designed stainless steel cell for $\mathrm{Hg}$ which can be refilled without breaking the vacuum. The flux of the latter cell is stable to within \pm 1.5 and $\pm 3 \%$ over a period of 2 and 30 hours, respectively. The $\mathrm{CdTe}$ and $\mathrm{Te}$ fluxes depend upon how long their respective shutters have been closed and/or open. For example the $\mathrm{Te}$ flux is much larger initially if the shutter has been closed for only a few minutes. It then reaches its steady state value after 5 to 10 minutes. Therefore we have used the following procedure in order to establish and measure the $\mathrm{CdTe}$ and Te fluxes used during the superlattice growth. The CdTe and Te shutters are opened and closed for the same periods of time used later during the growth. In order to insure stable fluxes, this is continued at least one hour up until the $\mathrm{Hg}$ cell is opened and the superlattice growth is started. This periodic opening and closing of the shutters has to be interrupted for about 20 seconds when growth begins, which causes the fluxes to deviate from their steady state values for the initial 20 to 30 seconds. The steady state values for $\mathrm{CdTe}, \mathrm{Te}$ and $\mathrm{Hg}$ were $3 \times 10^{-7}$, $6 \times 10^{-7}$ and $2.8 \times 10^{-4}$ Torr, respectively. In this article pressure is loosely referred to as flux. This $\mathrm{Hg}$ flux is roughly 2.5 times larger than the minimum flux necessary to maintain epitaxial growth of $\mathrm{HgTe}$.

The $\mathrm{Hg}_{1-x} \mathrm{Cd}_{x} \mathrm{Te}-\mathrm{HgTe}$ superlattices were grown on (001) $\mathrm{Cd}_{0.96} \mathrm{Zn}_{0.04} \mathrm{Te}$ substrates which had been degreased, chemo-mechanically polished for several minutes in a weak solution of bromine in methanol and then rinsed in methanol. Immediately prior to mounting the $5 \times 10 \times 1 \mathrm{~mm}$ substrates on a molybdenum holder with a solution of graphite in isopropanol and loading into the MBE system, they were rinsed in de-ionized water, briefly dipped in hydrochloric acid and then rinsed in de-ionized water so as to remove all of the original oxide and carbon from the substrate surface. ${ }^{13}$ We have found that the newly formed oxide resulting from this previous step is much more easily evaporated from the surface. ${ }^{14}$ This is accomplished by heating the substrates at temperatures up to about $320^{\circ} \mathrm{C}$ while monitoring the substrate surface by reflection high energy electron diffraction (RHEED) as described elsewhere. ${ }^{15}$ The substrate temperature was measured with an accuracy of $\pm 2{ }^{\circ} \mathrm{C}$ by means of a thermocouple which was in physical contact with a molybdenum substrate holder. The thermocouple was carefully calibrated at the melting point of indium.

Before the superlattice was grown, a thin CdTe buffer was grown on the $(001) \mathrm{Cd}_{0.96} \mathrm{Zn}_{0.04} \mathrm{Te}$ substrate at $270^{\circ} \mathrm{C}$ until the reflection high electron energy diffraction (RHEED) pattern indicated that the surface was smooth by the presence of short streaks. The thickness of this buffer was between 30 and $1000 \AA$. This surface was characterized by a $(2 \times 1)$ half order reconstruction in the [0 $0 \overline{1} 1]$ azimuth. We use the convention of referring to the direction of the incident electrons when referring to reconstruction in a particular azimuth. The superlattice was then grown at $180^{\circ} \mathrm{C}$.

\section{B. X-ray diffraction details and theory}

We have used a high resolution five crystal $x$-ray diffractometer to determine accurate values for the well thickness, the average superlattice period and the deviation from this average in the superlattice. The $\mathrm{Cu} \mathrm{K} \alpha_{1}$ radiation was resolved by means of the Ge (220) Bragg reflection in a four crystal monochromator. The reasons for a measurable (002) Bragg reflection for the zinc blende structure as opposed to the diamond structure where it is forbidden is discussed by Möller et al. ${ }^{11}$ The rather large (002) Bragg reflection in these superlattices is caused primarily by the $\mathrm{HgTe}$ well; the structure factor for the (002) Bragg reflection is much larger for $\mathrm{HgTe}$ than for CdTe. ${ }^{10,11}$ To our knowledge this behavior, the large difference in the size of the structure factor between $\mathrm{HgTe}$ and $\mathrm{CdTe}$, is unique to this system and perhaps to related $\mathrm{Hg}$ containing superlattices. This is due to the relatively large size of the $\mathrm{Hg}$ atom with its' large number of electrons. In fact the structure factor goes to zero for $\mathrm{Hg}_{1-x} \mathrm{Cd}_{x} \mathrm{Te}$ with an $x$ value of about 0.88 . Thus an observable envelope of the superlattice satellites is due to the slit function corresponding to the $\mathrm{HgTe}$ layers. From the angular separation of the first order zero points of this envelope, $\Delta \omega_{\text {Z.P. }}$, we can calculate the average thickness of the HgTe well, $\bar{t}_{w}$, with an accuracy of $\geqslant \pm 2 \AA$, which depends on the number of satellites and the position of the first order zero points, $\Delta \omega_{Z . P .}$, relative to the satellites. Similarily the average superlattice period, $\bar{t}_{P}$, can be determined from the angular separation of the superlattice satellites, $\Delta \omega_{P}$. From the increased broadening of the higher order satellites, $\delta \omega_{\text {Sat. }}$, for the $(004)$ reflection, the deviation of the superlattice period from its average value throughout the structure can be obtained. Here we have used the following equations: ${ }^{16}$

$$
\begin{aligned}
& \bar{t}_{P}=\frac{\lambda\left|\gamma_{H}\right|}{\Delta \omega_{P} \sin \left(2 \theta_{B}\right)} \\
& \frac{\delta t_{P}}{\bar{t}_{P}}=\frac{\delta \omega_{\text {Sat. }} \bar{t}_{P} \sin \left(2 \theta_{B}\right)}{\lambda\left|\gamma_{H}\right|} \\
& \bar{t}_{w}=\frac{2 \cdot \lambda\left|\gamma_{H}\right|}{\Delta \omega_{Z . P .} \sin \left(2 \theta_{B}\right)}
\end{aligned}
$$

where $\lambda$ is the wavelength of the reflected $\mathrm{x}$-rays, $\theta_{B}$ is the Bragg angle for the substrate, $\gamma_{H}$ is $\mathbf{k}_{H} \times \mathbf{n}, \mathbf{k}_{H}$ is the scattered unit wavevector and $\mathbf{n}$ is the unit vector normal to the surface.

\section{Transmission electron microscopy}

Four superlattices, Q250(a,b,c,d), with an extremely short period were grown simultaneously. One of these short period superlattices, Q250a, was characterized by TEM and high resolution transmission electron microscopy. ${ }^{17}$ The as grown superlattice was cut along a 110 plane of the (001) structure. Small pieces were glued face to face with epoxy resin and then thinned in order to insure transparency to electrons first by mechanical means and thereafter by means of $\mathrm{Ar}$ ion milling on a liquid ni- 


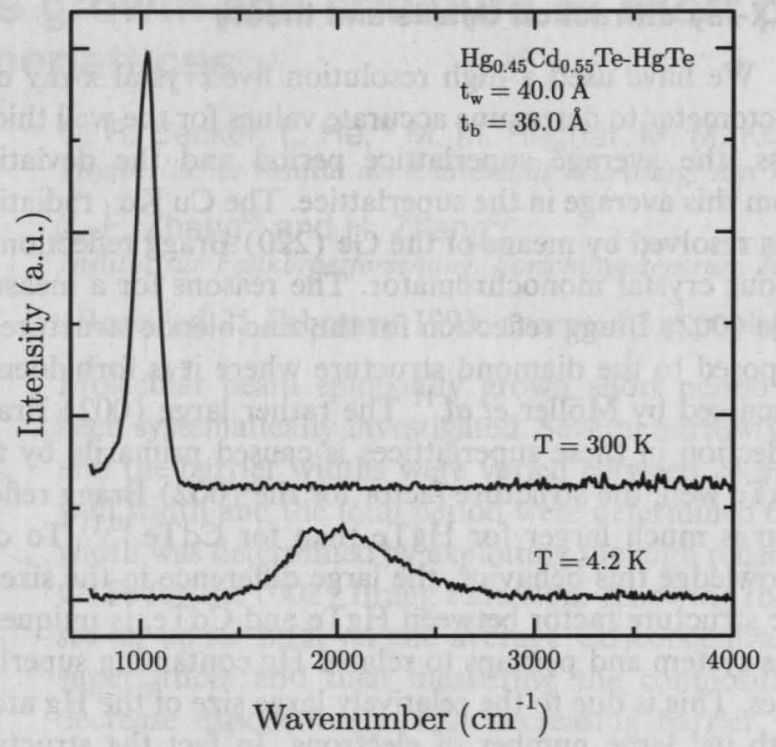

FIG. 1. Infrared photoluminescence spectra of the $\mathrm{Hg}_{0.45} \mathrm{Cd}_{0.55} \mathrm{Te}-\mathrm{HgTe}$ superlattice Q214 at 4.2 and $300 \mathrm{~K}$.

trogen cooled stage. These cross-section TEM samples were examined with a JEOL 4000EX transmission electron microscope operated at $400 \mathrm{kV}$.

\section{Composition of wells and barriers}

In order to determine the composition of our barriers, we have grown alloys with the same $\mathrm{CdTe}$ and $\mathrm{Hg}$ fluxes and at the same temperature used for the superlattice. In addition we have grown alloys with periodic growth stops of the same duration as that required for the growth of the $\mathrm{HgTe}$ well. In other words the growth conditions including the opening and closing of the $\mathrm{Te}$ and $\mathrm{CdTe}$ shutters were identical, with the exception of no Te flux. We determined the composition of the alloys grown by both of these methods from the $\mathrm{E}_{0}{ }^{18}$ and $\mathrm{E}_{1}$ (L6-L4,5) ${ }^{19,20}$ energy gaps by means of transmission and reflection measurements, respectively, to be $0.69 \pm 0.02$. However one condition, the presence of the $\mathrm{HgTe}$ wells, is not the same and interdiffusion in these short period superlattices must be taken into consideration.

In order to set an upper limit on the average barrier composition, $\bar{x}_{b}$, annealing experiments were carried out on several superlattices at 240 to $250^{\circ} \mathrm{C}$ for 24 hours. The superlattice was placed in a quartz ampoule together with either a drop of $\mathrm{Hg}$ which could not come into contact with the superlattice or with 400 mbar of $99.999 \%$ pure Ar gas. In the latter case, the superlattice surface was held in contact with a clean CdTe substrate by means of a weak tantalum spring on a piece of molybdenum.

\section{RESULTS AND DISCUSSION}

\section{A. Photoluminescence measurements}

Fourier transform infrared luminescence spectra of one of the superlattices at temperatures of 4.2 and $300 \mathrm{~K}$ are shown in Fig. $1 .^{12}$ The photoluminescence spectra of

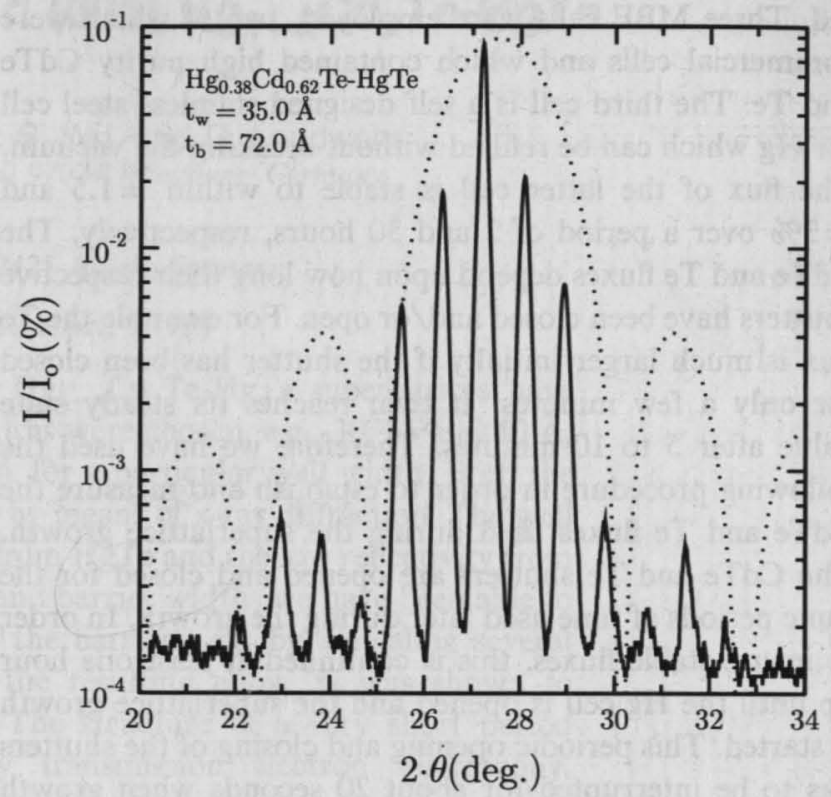

FIG. 2. X-ray rocking curve for the (002) Bragg reflection from the $\mathrm{Hg}_{0.38} \mathrm{Cd}_{0.62} \mathrm{Te}-\mathrm{HgTe}$ superlattice Q230. The full line represents the experimental data and the dotted line is the envelope of the superlattice satellites which is due to the slit function corresponding to the $\mathrm{HgTe}$ layers. The large number of satellites is an indication of the excellent structural quality of the superlattice.

almost all of the superlattices in this investigation consist of one nearly symmetric line. The full width at half maximum of this line lies between 16 and $60 \mathrm{meV}$ at $4.2 \mathrm{~K}$ and between 52 and $113 \mathrm{meV}$ at $300 \mathrm{~K}$. This attests to the good quality of these superlattices. These measurements as well as experimentally and theoretically determined absorption coefficients will be discussed in detail in a future publication.

\section{B. X-ray diffraction measurements}

A smoothed rocking curve of the $(002)$ reflection for a $\mathrm{Hg}_{1-x} \mathrm{Cd}_{x} \mathrm{Te}-\mathrm{HgTe}$ superlattice and the slit function used for the $\mathrm{HgTe}$ well thickness determination are shown in Fig. 2 as the full and dotted lines, respectively. The large number of satellites, which is typical of most of the investigated superlattices, is an indication of the high structural quality of these superlattices. The period, well and barrier thicknesses of all the superlattices investigated are listed in Table I. The variation of these periods from their average values, according to Eq. (2), is less than $10 \%$. This is also an indication of the good uniformity of these superlattices. The experimental uncertainty in the period and the $\mathrm{HgTe}$ well thickness is \pm 0.5 and $\geqslant \pm 2 \AA$, respectively.

Two of the extremely short period superlattices mentioned above, Q250a and Q250c, were investigated by x-ray diffraction in greater detail. From the measured rocking curve of the (002) Bragg reflection for Q250a shown in Fig. 3, we obtained an average superlattice period of 31.4 $\AA$, an average well thickness of $11.4 \AA$ and a total intensity for the zero order satellite of $3.1724 \times 10^{-6}$. The rocking curve for Q250c is nearly identical and consequently, so 
TABLE I. The number of periods, the period $(\AA)$, thickness $(\AA)$ and average Cd concentration of both the well and barrier for the investigated superlattices. Both measured values of the Cd concentration for the barriers, $\bar{x}_{b}$, and empirical values for $\bar{x}_{b}$ and $\bar{x}_{w}$, which were calculated by using the Cd concentration profile according to Ref. 8, are included.

\begin{tabular}{|c|c|c|c|c|c|c|c|}
\hline & Periods & $\begin{aligned} & \bar{t}_{P} \\
\pm & 0.5\end{aligned}$ & $\begin{array}{l}\bar{t}_{w} \\
\pm 2\end{array}$ & $\begin{array}{l}\bar{t}_{b} \\
\mp 2\end{array}$ & $\bar{x}_{w}$ & empirical & $\begin{array}{c}\bar{x}_{b} \\
\text { experimental }\end{array}$ \\
\hline Q250 & 900 & 31.4 & 11.4 & 20.0 & 0.04 & 0.44 & $0.36 \pm 0.03$ \\
\hline Q211 & 100 & 47.0 & 16.0 & 31.0 & 0.03 & 0.53 & $0.50 \pm 0.03$ \\
\hline Q178 & 90 & 54.1 & 30.6 & 23.5 & 0.02 & 0.48 & \\
\hline Q218 & 100 & 64.9 & 32.2 & 32.7 & 0.02 & 0.54 & $0.55 \pm 0.03$ \\
\hline Q168 & 180 & 67.4 & 29.6 & 37.8 & 0.02 & 0.56 & \\
\hline Q247 & 100 & 82.5 & 31.4 & 51.1 & 0.02 & 0.59 & \\
\hline Q215 & 100 & 120.8 & 30.7 & 90.1 & 0.02 & 0.64 & \\
\hline Q174 & 90 & 121.0 & 30.3 & 90.7 & 0.02 & 0.64 & $0.64 \pm 0.02$ \\
\hline Q167 & 140 & 87.0 & 35.1 & 51.9 & 0.01 & 0.59 & \\
\hline Q230 & 100 & 107.0 & 35.0 & 72.0 & 0.01 & 0.62 & \\
\hline Q214 & 100 & 76.0 & 40.0 & 36.0 & 0.01 & 0.55 & \\
\hline Q200 & 100 & 77.1 & 38.4 & 38.7 & 0.01 & 0.56 & · \\
\hline Q195 & 100 & 82.0 & 38.7 & 43.3 & 0.01 & 0.58 & \\
\hline Q163 & 100 & 89.0 & 40.0 & 49.0 & 0.01 & 0.59 & $0.60 \pm 0.02$ \\
\hline Q204 & 20 & 96.8 & 38.9 & 56.9 & 0.01 & 0.61 & \\
\hline Q164 & 80 & 116.1 & 43.0 & 73.1 & 0.01 & 0.62 & \\
\hline Q171 & 90 & 129.1 & 43.5 & 85.6 & 0.01 & 0.63 & \\
\hline Q162 & 100 & 80.0 & 47.9 & 32.1 & 0.01 & 0.54 & \\
\hline Q165 & 100 & 106.0 & 70.0 & 36.0 & 0.01 & 0.55 & \\
\hline
\end{tabular}

are resulting average values for the period and the well thickness, i.e., 31.4 and $11.4 \AA$. The simulation of Q250a shown in Fig. 3 was calculated using a concentration profile across the $\mathrm{Hg}_{1-x} \mathrm{Cd}_{x} \mathrm{Te}-\mathrm{HgTe}$ interface with the same width and shape as the interface according to Kim et al. ${ }^{8}$

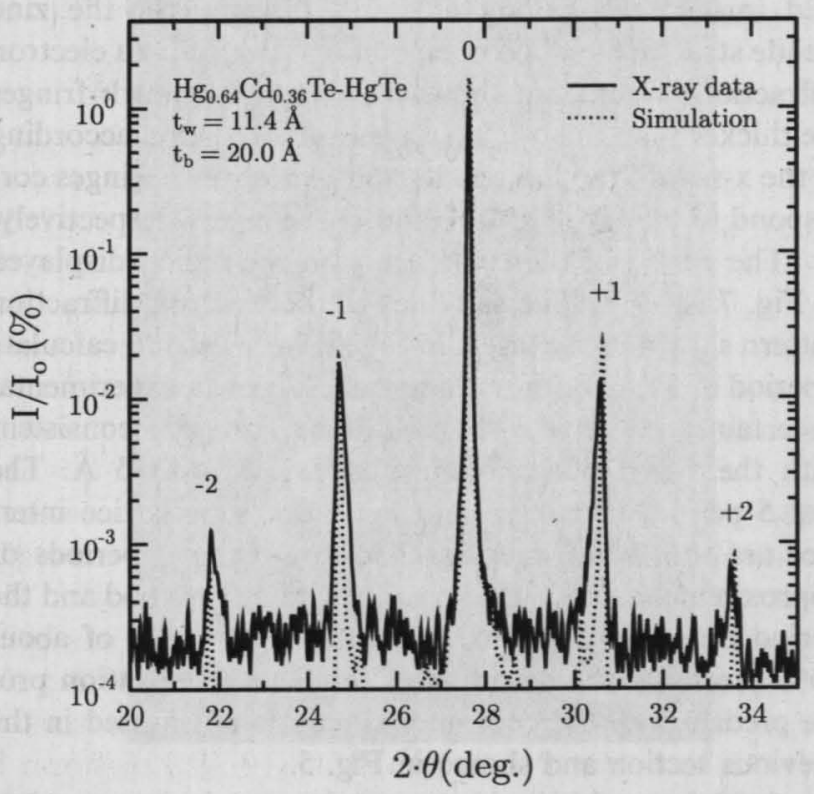

FIG. 3. X-ray rocking curve for the $(002)$ Bragg reflection from the $\mathrm{Hg}_{0.64} \mathrm{Cd}_{0.36} \mathrm{Te}-\mathrm{HgTe}$ superlattice Q250a. The full line with random noise represents the experimental data and the dotted line is a simulation of the data.
This concentration profile is shown in Fig. 4. If an abrupt interface was employed then the simulated intensities of the satellites were much larger, e.g., the second order satellites were one to two orders of magnitude larger. Furthermore, if an interface with approximately twice the width of the published interface was used, then the second

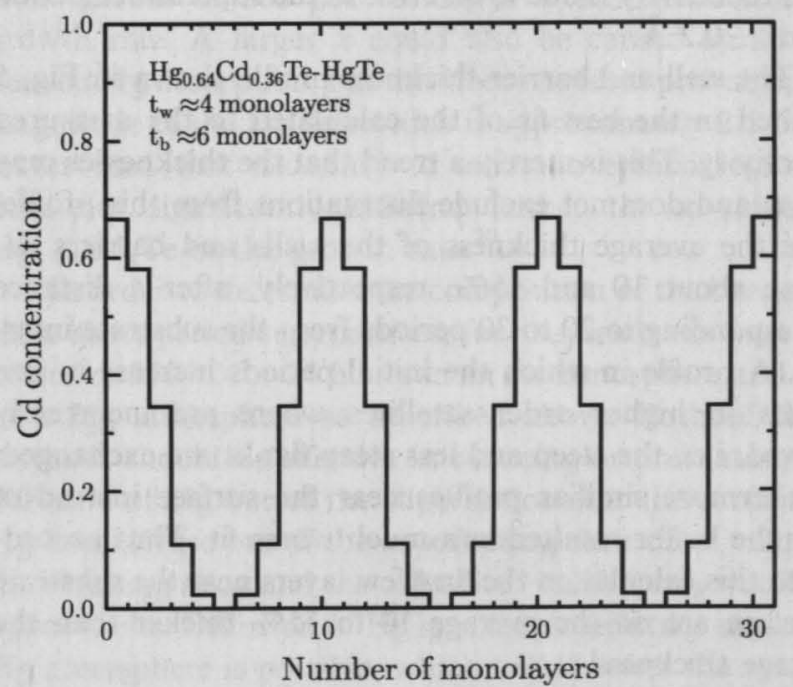

FIG. 4. Cd concentration profile used in the x-ray simulation and in the empirical determination of the average Cd barrier concentration, $\bar{x}_{b}$, for Q250a. The width and shape of the $\mathrm{Hg}_{0.64} \mathrm{Cd}_{0.36} \mathrm{Te}-\mathrm{HgTe}$ interface is the same as the experimental width and shape according to Ref. 8. 


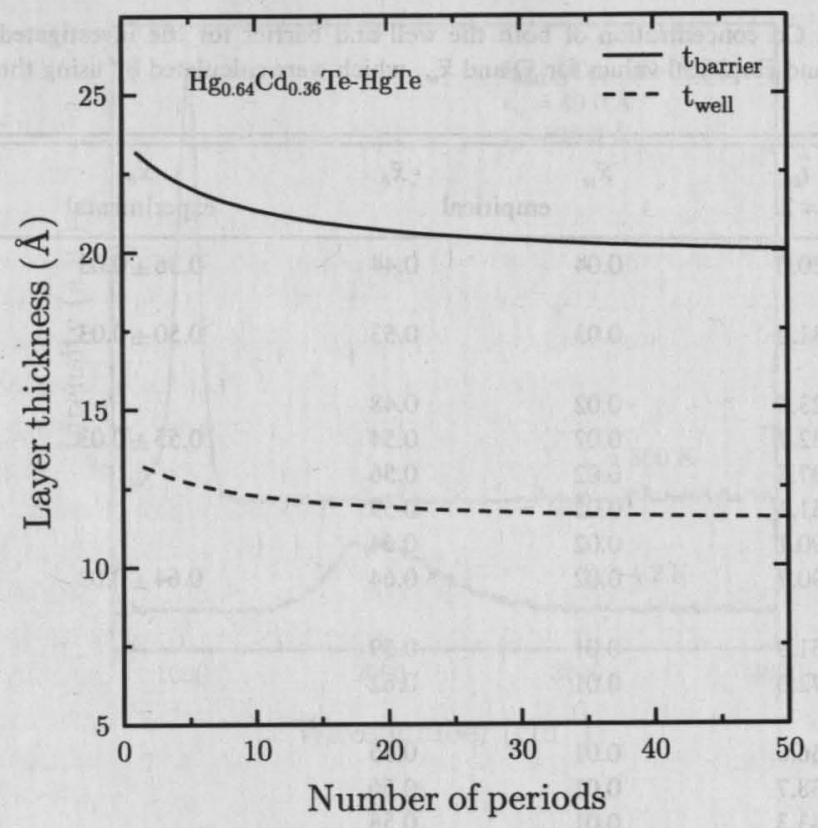

FIG. 5. Possible thickness profiles starting at the superlattice/substrate interface for both the wells and barriers in the $\mathrm{Hg}_{0.64} \mathrm{Cd}_{0.36} \mathrm{Te}-\mathrm{HgTe}$ superlattice Q250a which result from the intensity simulation shown in Fig. 3.

order satellites were no longer discernible. Thus according to these simulations, the concentration profile across the $\mathrm{Hg}_{1-x} \mathrm{Cd}_{x} \mathrm{Te}-\mathrm{HgTe}$ interface in these superlattices is similar to that of the interface according to Kim et al. ${ }^{8}$

In order to fit the shape of the superlattice satellites we manipulated the thickness profile of the well and barrier by multiplying a constant well and barrier thickness with a hyperbolic function. The resulting profile was used in a dynamic calculation of the reflectivity, which was fit to the measured rocking curve with the intensity and the shape of the satellites as criteria for this comparison. The small misfit in the satellite positions of the experimental and simulated reflectivity is due to an error in the superlattice period $\delta t_{P}$ of $\approx 0.2 \AA$.

The well and barrier thickness profile shown in Fig. 5 resulted in the best fit of the calculated to the measured reflectivity. This is merely a trend that the thicknesses may follow and does not exclude fluctuations from this profile. Here the average thickness of the wells and barriers decrease about 10 and $15 \%$, respectively, after a distance corresponding to 20 to 30 periods from the substrate interface. A profile in which the initial periods increase in size results in higher order satellites where are incorrectly skewed, i.e., the steep and less steep flanks are exchanged. Furthermore similiar profiles near the surface instead of near the buffer resulted in a much worse fit. Thus according to this calculation the first few layers near the substrate interface are on the average 10 to $15 \%$ thicker than the average thickness.

The fit of the simulated intensity to the measured total intensity of the zero order satellite yielded an average $\mathrm{Cd}$ content for the whole structure of $\bar{x} \approx 0.24 \pm 0.05$. The calculated thickness profile fits the experimental rocking

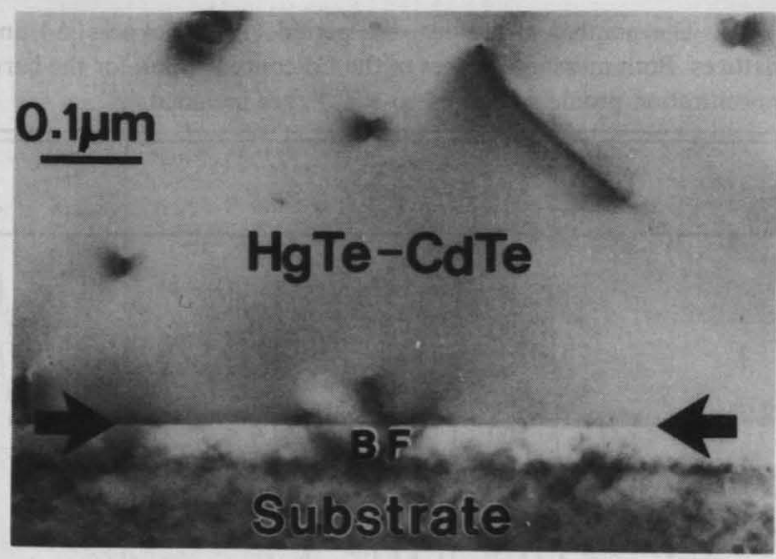

FIG. 6. The morphology of a cross-section of the superlattice Q250a near the superlattice/buffer and buffer/substrate interfaces is shown in a low magnification TEM image. The $30 \AA$ thick CdTe buffer is indicated by the letters BF and the interface by two bold arrows.

curve best with a quantum well concentration of $\bar{x}_{w} \approx 0.09 \pm 0.05$ and a barrier concentration of $\bar{x}_{b} \approx 0.33 \pm 0.05$. These values are in reasonable agreement with the results of the annealing experiment on Q250a which are discussed below and are summarized in Table I.

\section{Transmission electron microscopy}

The morphology of a cross-section of Q250a near the superlattice/buffer and buffer/substrate interfaces is shown in a low magnification TEM image in Fig. 6. The TEM results presented here are dealt with in much greater detail by Zhang et al. ${ }^{17}$ The $30 \AA$ thick CdTe buffer is indicated by the letters $\mathrm{BF}$ and the interface by two bold arrows. The buffer/substrate interface is rough and not clearly defined while, as might be expected, a smooth and sharp interface is present between the superlattice and the buffer. A bright field image taken along a $[1, \overline{1}, 0]$ direction of the zinc blende structure and a corresponding selected area electron diffraction pattern are shown in Fig. 7. The dark fringes are thicker than the bright fringes and therefore, according to the $\mathrm{x}$-ray diffraction results, dark and bright fringes correspond to the $\mathrm{Hg}_{1-x} \mathrm{Cd}_{x} \mathrm{Te}$ and $\mathrm{HgTe}$ layers, respectively.

The period of the superlattice in the region displayed in Fig. 7 is $33 \AA$. The satellites in the electron diffraction pattern shown in the insert in Fig. 7, were used to calculate a period of $34 \AA$. Both of these values have an experimental uncertainty of about $\pm 3 \AA$ and are therefore consistent with the $x$-ray diffraction results, i.e., $31.4 \pm 0.5 \AA$. The first 5 periods starting from the buffer/superlattice interface are somewhat irregular. However after 5 periods or approximately $150 \AA$, the structural quality is good and the period is regular. The experimental uncertainty of about $10 \%$ precludes the detection of the $\mathrm{Cd}$ concentration profile predicted by the computer simulation discussed in the previous section and shown in Fig. 5.

A high resolution electron microscopic image taken along the $[1, \overline{1}, 0]$ direction is displayed in Fig. 8 . The brighter $\mathrm{HgTe}$ and darker $\mathrm{Hg}_{0.64} \mathrm{Cd}_{0.36} \mathrm{Te}$ layers can be more easily distinguished along the $(001)$ by viewing from 


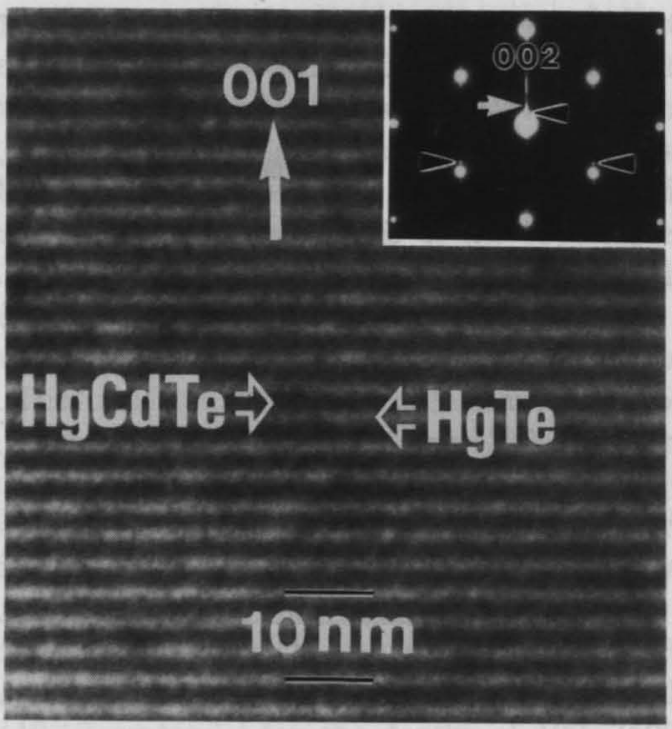

FIG. 7. A bright field image taken along a $[1, \overline{1}, 0]$ direction of the zinc blende structure and a corresponding selected area electron diffraction pattern. The dark fringes are thicker than the bright fringes and therefore, according to the $\mathrm{x}$-ray diffraction results, dark and bright fringes correspond to the $\mathrm{Hg}_{0.64} \mathrm{Cd}_{0.36} \mathrm{Te}$ and $\mathrm{HgTe}$ layers, respectively. The first and second order satellites along the [001]* direction are indicated in the insert by black and white arrows, respectively.

the side at a glancing angle. The contrast between these layers is produced by a $\mathrm{Cd}$ concentration difference of 0.36 or less, according to the results of the annealing experiment which is discussed in the next section. The

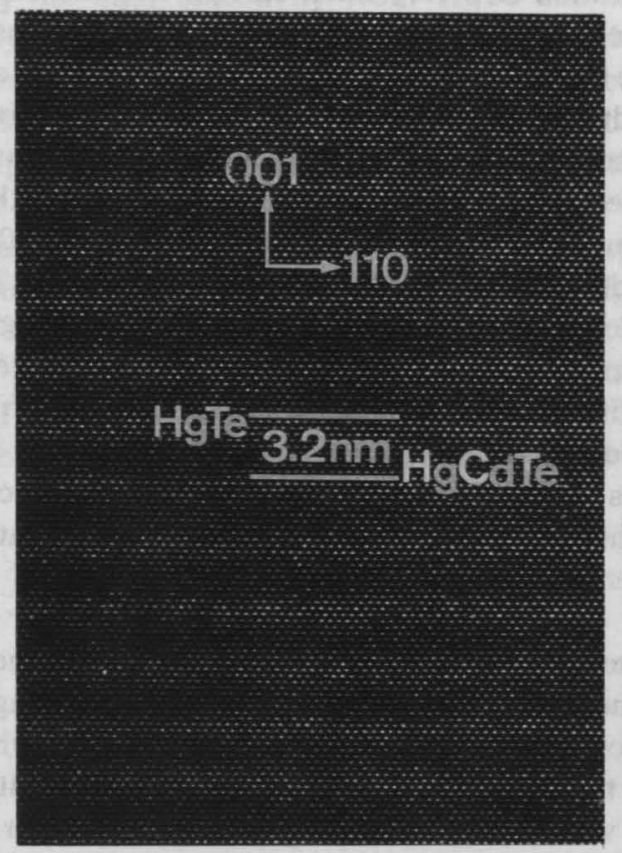

FIG. 8. A high resolution electron microscopic image taken along the $[1, \overline{1}, 0]$ direction. The brighter $\mathrm{HgTe}$ and darker $\mathrm{Hg}_{0.64} \mathrm{Cd}_{0.36} \mathrm{Te}$ layers can be more easily distinguished along the $(001)$ by viewing from the side at a glancing angle. The contrast between these layers is produced by an average $\mathrm{Cd}$ concentration difference of $36 \%$ or less.
$\mathrm{HgTe} / \mathrm{Hg}_{0.64} \mathrm{Cd}_{0.36} \mathrm{Te}$ interfaces display appreciable waviness as might be expected for such narrow layers.

\section{Composition of wells and barriers}

Prior to approximately 1986 the composition of the well and barrier material in $\mathrm{Hg}_{1-x} \mathrm{Cd}_{x} \mathrm{Te}-\mathrm{HgTe}$ superlattices was assumed to be $\mathrm{HgTe}$ and $\mathrm{CdTe}$, respectively. In 1987 Reno et al. ${ }^{21}$ determined the $x$ value of a thin layer, i.e., $150-170 \AA$ of $(001) \mathrm{Hg}_{1-x} \mathrm{Cd}_{x} \mathrm{Te}$ barrier material grown at $185^{\circ} \mathrm{C}$ to be 0.85 by $\mathrm{x}$-ray photoelectron spectroscopy. Due to absorption of the photo-emitted electrons, this is the $x$ value for approximately $50 \AA$ of the layer near the surface. Later Schulman et al. ${ }^{9}$ determined the $x$ value of a test layer of $(001) \mathrm{Hg}_{1-x} \mathrm{Cd}_{x} \mathrm{Te}$ grown at $175^{\circ} \mathrm{C}$ to be 0.85 . Most of the recent literature either assumes this $x$ value to be correct, independent of the substrate temperature and the other growth parameters, or does not mention how the $x$ value is determined. A recent exception is the work of Monterrat et al. ${ }^{22}$ which reports an $x$ value of approximately 0.70 for barrier material in (001) single and multiple quantum wells grown at $180^{\circ} \mathrm{C}$.

As mentioned above, the composition of alloys grown with the same growth parameters as those used for our barrier material was determined to be $0.69 \pm 0.02$. We found that MBE growth with the same growth conditions as described above but with illumination of the substrate with an $\mathrm{Ar}$ ion laser, resulted in an $x$ value of 0.85 . Wu et al. ${ }^{15}$ have shown that illumination with an $\mathrm{Ar}$ ion laser as well as irradiation with the high energy electrons used in their RHEED observations, i.e., $10 \mathrm{keV}$, significantly reduces the desorption time for excess $\mathrm{Te}$ from a $\mathrm{Te}$ stabilized CdTe surface. Because the $x$ value of $\mathrm{Hg}_{1-x} \mathrm{Cd}_{x} \mathrm{Te}$ is governed primarily by the substrate temperature and the $\mathrm{CdTe}$ to Te flux ratio, ${ }^{20}$ an enhanced desorption of $\mathrm{Te}$ from $\mathrm{Hg}_{1-x} \mathrm{Cd}_{x} \mathrm{Te}$ would result in a larger $x$ value. This is consistent with our observations from RHEED oscillations that the growth rate for $\mathrm{Hg}_{0.3} \mathrm{Cd}_{0.7} \mathrm{Te}$ and $\mathrm{HgTe}$ decreases with increasing high energy electron intensity, i.e., an electron current of $200 \mu \mathrm{A}$ results in a $10 \%$ reduction in growth rate. A larger $x$ could also be caused by an enhanced $\mathrm{Hg}$ desorption, but this does not seem probable; the magnitude of the $\mathrm{Hg}$ flux which is approximately 2.5 times larger than that necessary to maintain epitaxial growth does not significantly influence either the $x$ value of $\mathrm{Hg}_{1-x} \mathrm{Cd}_{x} \mathrm{Te}$ or the growth rate. ${ }^{20}$

In order to determine the composition of the barrier in these short period superlattices, four superlattices were annealed at $250^{\circ} \mathrm{C}$ for 24 hours in an $\mathrm{Ar}$ atmosphere and one in a $\mathrm{Hg}$ atmosphere as described above. Both of these methods should be effective in reducing or preventing the diffusion of $\mathrm{Hg}$ out of the superlattice near the surface. If $\mathrm{Hg}$ does diffuse out of the superlattice, then the $x$ value of the resulting alloy and the calculated value for $\bar{x}_{b}$ would be upper limits. Diffusion of $\mathrm{Hg}$ into the superlattice under an $\mathrm{Hg}$ atmosphere is possible, which would lower the $x$ value near the surface.

The $x$ value of one of the resulting alloys was determined from reflection measurements of the $E_{1}$ gap to be $0.48 \pm .01$. The corresponding $x$ value from transmission 


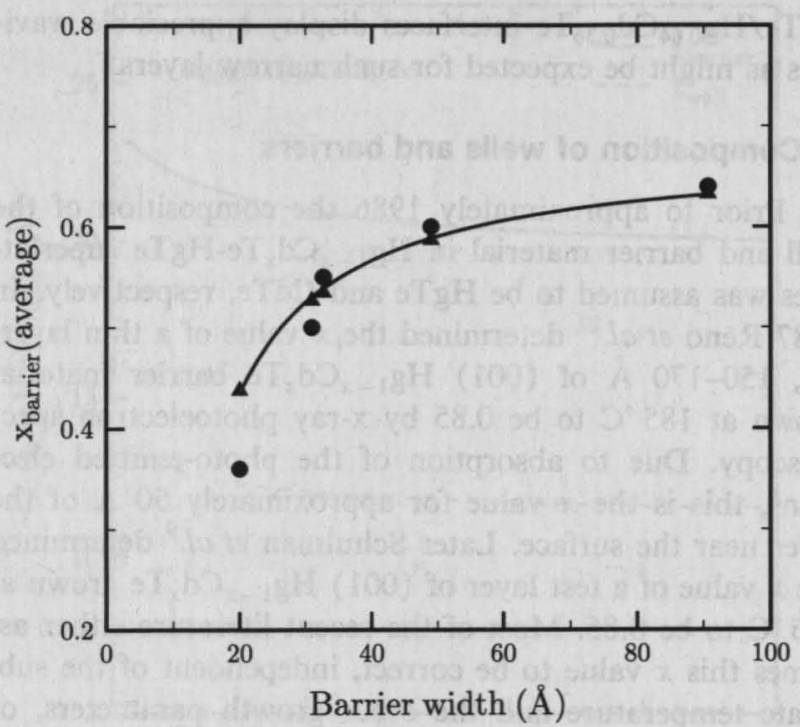

FIG. 9. $\bar{x}_{b}$ as a function of the barrier width is shown as filled circles. The filled triangles represent values which have been calculated using the $\mathrm{Cd}$ concentration profile published in Ref. 8. The curve is a least square fit of an exponential function to the calculated data, see Eq. (4).

measurements of the $\mathrm{E}_{0}$ gap is somewhat higher, i.e., 0.52 . Furthermore the discrepancy between the $\mathrm{E}_{0}$ and the $\mathrm{E}_{1}$ gap increases with decreasing superlattice thickness. This is an indication that diffusion between the CdTe substrate and the $\mathrm{Hg}_{1-x} \mathrm{Cd}_{x} \mathrm{Te}$ alloy is responsible for this discrepancy. Reflection measurements should be less sensitive to changes in $x$ due to diffusion near the substrate/alloy interface. Therefore $x$ values as determined from reflection measurements of the $E_{1}$ gap are used in the following with one exception. The exception is the superlattice which was annealed in an $\mathrm{Hg}$ atmosphere and could consequently have a lower $x$ value near the surface. In view of the uncertainty in this case, an average of the two $x$ values (i.e., 0.24 and 0.22 for the $E_{0}$ and $E_{1}$ gap, respectively) is employed.

If all of the CdTe is in the barrier whose width is $91 \pm 2$ $\AA$, then $\bar{x}_{b}$ is $0.64 \pm 0.02$. Obviously, this is an upper limit for $\bar{x}_{b}$ due to the assumption that all of the CdTe is in the barrier. Interdiffusion between the well and the barrier becomes more prominent as the well and barrier become thinner. The $\bar{x}_{b}$ upper limit for these samples are plotted as a function of the barrier width in Fig. 9 and are listed in Table I. As can be seen, $\bar{x}_{b}$ is appreciably lower for narrower barriers.

As mentioned above, Kim et al. ${ }^{8}$ have experimentally determined the concentration profile in a similar superlattice which was also grown at $180^{\circ} \mathrm{C}$. By assuming that the width and shape of the interfaces in this investigation are the same (see Fig. 4), as suggested by the satellite intensity simulations which were discussed in the section on $\mathrm{x}$-ray diffraction, we have calculated values for $\bar{x}_{b}$, which are displayed as triangles in Fig. 9. The boundaries of the barriers were consistently chosen to occur at an $x$ value of 0.16 so as to ensure a better fit to the experimental data for the wider barriers. The fit of these calculated values to the

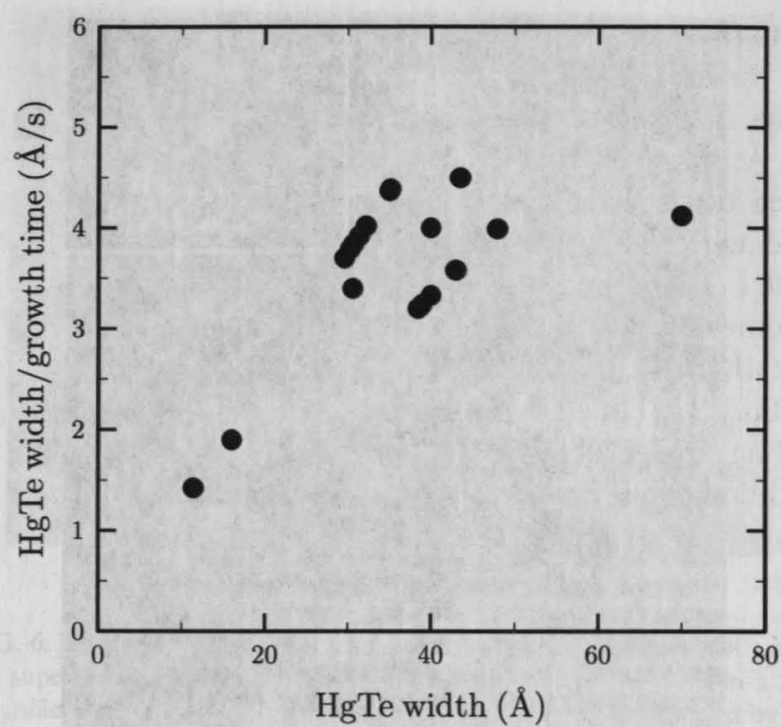

FIG. 10. The effective quantum well growth rate, quantum well width divided by the growth time, is plotted versus quantum well width.

experimental data is reasonable with a slightly worse fit for narrow barriers. This is to be expected when the barrier width begins to approach a value which is twice the width of the interface. The calculated values for both $\bar{x}_{b}$ and $\bar{x}_{w}$ are listed in Table I. The following empirical relationship is the result of a fit of an exponential function to the calculated values of $\bar{x}_{b}$ and the barrier widths:

$$
\bar{x}_{b}=x_{0}\left(2-e^{\left(6.4 / \bar{t}_{b}\right)}\right)
$$

where $\bar{t}_{b}$ is the barrier thickness in $\AA$ and $x_{0}$ is the $\mathrm{Cd}$ concentration of a very thick barrier, i.e., the $\mathrm{Cd}$ concentration of alloys grown under the same conditions.

The effective quantum well growth rate, i.e., quantum well width divided by the growth time, is plotted in Fig. 10 as a function of well width for all of the superlattices investigated. As can be seen, this effective growth rate is significantly smaller for narrower wells, i.e., $\leqslant 20 \AA$. The well width of the corresponding two superlattices is appreciably less than expected. This can be explained by the increasing relative importance of diffusion with decreasing well width. In other words, the difference between the concentration profile mentioned above and an abrupt interface becomes more important when the well width is comparable to the width of the interface. If we assume that initially the interfaces are abrupt and that all of the $\mathrm{Cd}$ is in the barrier, then the initial quantum well is wider and the quantum well growth rate of these two superlattices is 2.8 $\AA / \mathrm{s}$. These corrected growth rates are in better agreement with those of the other superlattices. It is worthwhile mentioning that the quantum well $(\mathrm{HgTe})$ growth rate for the thicker wells is 60 to $80 \%$ larger than the growth rate of a thick epilayer of $\mathrm{HgTe}$ under the same conditions.

One of the superlattices, Q250b, with the extremely short period of $31.4 \AA$ was annealed at $250^{\circ} \mathrm{C}$ for 24 hours under a $\mathrm{Hg}$ atmosphere. The $x$ value of the resulting alloy was determined from transmission and reflection measure- 
ments to be 0.24 and 0.22 , respectively. If all of the CdTe is in the barrier whose width is $20.0 \AA$ then the $x$ value of the barrier is about 0.36 . For this narrow well width, i.e., $11.4 \AA$; however, this is not a realistic assumption as suggested by the calculated value for $\bar{x}_{w}$, i.e., 0.04 , which is listed together with values for all of the superlattices in Table I. Therefore the average $x$ value in the barrier should be somewhat less than 0.36 .

Obviously appreciable interdiffusion has taken place in Q250b. The initial barrier composition, $\bar{x}_{b}$, was 0.69 according to the alloys grown under nearly identical growth conditions. This superlattice consists of 900 periods each with a width of $31.4 \AA$ which were subjected to the growth temperature of $180^{\circ} \mathrm{C}$ for 4 hours. As mentioned above Kim et $a l^{8}$ have shown that interdiffusion is larger near the surface. Their published values for the $\mathrm{Hg}$ diffusion constant at $180^{\circ} \mathrm{C}$ are approximately $1 \times 10^{-17}, 1 \times 10^{-18}$ and $1 \times 10^{-19} \mathrm{~cm}^{2} \mathrm{sec}^{-1}$ for the depths of 100,3500 and $7000 \AA$ respectively. The time spent at a distance of $3500 \AA$ or less from the surface was 30 minutes. Thus to a first approximation, a diffusion length for $\mathrm{Hg}$ of $\sqrt{D t} \approx 13 \AA$ can be expected. This is roughly the barrier width and therefore a reduction in $\bar{x}_{b}$ from 0.69 to 0.36 or less is reasonable. Furthermore the calculated value for $\bar{x}_{b}$ using the published $\mathrm{Cd}$ concentration profile ${ }^{8}$ for a similar superlattice is in reasonable agreement with the experimental value, see Fig. 9.

\section{CONCLUSIONS}

Both the well width as well as the total period of a number of MBE grown short period (001) $\mathrm{Hg}_{1-x} \mathrm{Cd}_{x} \mathrm{Te}-\mathrm{HgTe}$ superlattices were determined directly by $\mathrm{x}$-ray diffraction. The well width was determined by exploiting the high reflectivity from $\mathrm{HgTe}$ and the low reflectivity from CdTe for the (002) Bragg reflection. An upper limit for the average composition of the barriers, $\bar{x}_{b}$, was determined for the barriers. $\bar{x}_{b}$ was found to decrease exponentially with decreasing barrier width, e.g., $\bar{x}_{b} \approx 0.64$ and 0.36 for barrier widths of 90.7 and $20.0 \AA$ respectively. This exponential dependence can be explained by assuming that the width and shape of the $\mathrm{Hg}_{1-x} \mathrm{Cd}$ ${ }_{x} \mathrm{Te} / \mathrm{HgTe}$ interface of all the investigated superlattices are identical to an experimentally determined concentration profile. ${ }^{8}$ The relative importance of this interface, i.e., interdiffusion between the barrier and well, increases with deceasing barrier width. The structure of a very short period superlattice, i.e., $31.4 \AA$ was also investigated by transmission electron microscopy, corroborating the $\mathrm{x}$-ray diffraction results.

\section{ACKNOWLEDGMENTS}

The support of this project by the Bundesministerium für Forschung und Technologie (contract number TK 0369), the Deutsche Forschungsgemeinschaft and the Alexander von Humboldt-Stiftung is gratefully acknowledged.

${ }^{1}$ J. N. Schulman and T. C. McGill, Appl. Phys. Lett. 34, 663 (1979).

${ }^{2}$ J. P. Faurie, A. Million, and J. Piaguet, Appl. Phys. Lett. 41, 713 (1982).

${ }^{3}$ J. P. Faurie, IEEE J. Quantum Electron. QE-22, 1656 (1986).

${ }^{4}$ T. C. McGill, G. Y. Wu, and S. R. Hetzler, J. Vac. Sci. Technol. A 4, 2091 (1986).

${ }^{5}$ J. R. Meyer, C. A. Hoffman, and F. J. Bartoli, Semicond. Sci. Technol. 5, S90 (1990).

${ }^{6}$ D. L. Smith, T. C. McGill, and J. N. Schulman, Appl. Phys. Lett. 43, 180 (1983).

${ }^{7}$ J. Reno and J. P. Faurie, Appl. Phys. Lett. 49, 409 (1986).

${ }^{8}$ Y. Kim, A. Ourmazd, M. Bode, and R. D. Feldman, Phys. Rev. Lett. 63, 636 (1989).

${ }^{9}$ J. N. Schulman, O. K. Wu, E. A. Patten, J. W. Han, Y. Lansari, L. S. Kim, J. W. Cook, Jr., and J. F. Schetzina, Appl. Phys. Lett. 53, 2420 (1988).

${ }^{10}$ M. Möller, Doctoral Dissertation, Physikalisches Institut, University of Würzburg (1991).

${ }^{11}$ M. Möller, R. N. Bicknell-Tassius, and G. Landwehr, J. Appl. Phys. 72, 5108 (1992).

${ }^{12}$ M. M. Kraus, M. M. Regnet, C. R. Becker, R. N. Bicknell-Tassius, and G. Landwehr, J. Appl. Phys. 71, 5610 (1992).

${ }^{13}$ Y. S. Wu, C. R. Becker, A. Waag, R. N. Bicknell-Tassius, and G. Landwehr, Appl. Phys. Lett. 60, 1878 (1992).

${ }^{14}$ Y. S. Wu, C. R. Becker, A. Waag, R. Schmiedl, S. Einfeldt, and G. Landwehr, J. Appl. Phys. 73, 7385 (1993).

${ }^{15}$ Y. S. Wu, C. R. Becker, A. Waag, R. N. Bicknell-Tassius, and G. Landwehr, J. Appl. Phys. 69, 268 (1991).

${ }^{16}$ M. Quillec, Structural Characterization of Superlattices by X-Ray Diffraction, Springer Proceedings in Physics (Les Houches, France, 1986), Vol. 13, pp. $121 \mathrm{ff}$.

${ }^{17}$ X. F. Zhang, H. Zhang, L. He, C. R. Becker, and G. Landwehr (unpublished).

${ }^{18}$ G. L. Hansen, J. L. Schmit, and T. N. Casselman, J. Appl. Phys. 53, 7099 (1982).

${ }^{19}$ R. R. Galazka and A. Kisiel, Phys. Status Solidi 34, 63 (1969).

${ }^{20}$ L. He, C. R. Becker, R. N. Bicknell-Tassius, S. Scholl, and G. Landwehr, J. Appl. Phys. 73, 3305 (1993).

${ }^{21}$ J. Reno, R. Sporken, Y. J. Kim, C. Hsu, and J. P. Faurie, Appl. Phys. Lett. 51, 1545 (1987).

${ }^{22}$ E. Monterrat, L. Ulmer, R. Mallard, N. Magnea, and J. L. Pautrat, J. Appl. Phys. 71, 1774 (1992). 